\title{
Is Histologic Thrombus Composition in Acute Stroke Linked to Stroke Etiology or to Interventional Parameters?
}

(D). Goebel, (D) B.-J. Gaida, (DI. Wanke, (D) C. Kleinschnitz, (D) M. Koehrmann, (D) M. Forsting, (D) C. Moenninghoff, (D) A. Radbruch, and (D) A. Junker

\section{ABSTRACT}

BACKGROUND AND PURPOSE: Detailed insight into the composition of thrombi retrieved from patients with ischemic stroke by mechanical thrombectomy might improve pathophysiologic understanding and therapy. Thus, this study searched for links between histologic thrombus composition and stroke subtypes and mechanical thrombectomy results.

MATERIALS AND METHODS: Thrombi from 85 patients who had undergone mechanical thrombectomy for acute ischemic stroke between December 2016 and March 2018 were studied retrospectively. Thrombi were examined histologically. Preinterventional imaging features, stroke subtypes, and interventional parameters were re-analyzed. Statistical analysis was performed with the Kruskal-Wallis test, Mann-Whitney $U$ test, or Spearman correlation as appropriate.

RESULTS: Cardioembolic thrombi had a higher percentage of macrophages and a tendency toward more platelets than thrombi of large-artery atherosclerotic stenosis $(P=.021$ and .003$)$ or the embolic stroke of undetermined source $(P=.037$ and .099$)$ subtype. Thrombi prone to fragmentation required the combined use of contact aspiration and stent retrieval $(P=.021)$ and were associated with an increased number of retrieving maneuvers $(P=.001)$, longer procedural times $(P=.001)$, and a higher lymphocyte content $(P=.035)$.

CONCLUSIONS: We interpreted the higher macrophage and platelet content in cardioembolic thrombi compared with large-artery atherosclerotic stenosis or embolic stroke of undetermined source thrombi as an indication that the latter type might be derived from an atherosclerotic plaque rather than from an undetermined cardiac source. The extent of thrombus fragmentation was associated with a more challenging mechanical thrombectomy and a higher lymphocyte content of the thrombi. Thus, thrombus fragmentation not only might be caused by the recanalization procedure but also might be a feature of a lymphocyte-rich, difficult-to-retrieve subgroup of thrombi.

ABBREVIATIONS: ESUS = embolic stroke of undetermined source; $\mathrm{H \& E}=$ hematoxylin-eosin; $\mathrm{LAA}=$ large-artery atherosclerotic stenosis; MT = mechanical thrombectomy; $\mathrm{mTICI}=$ modified $\mathrm{TICl} ; \mathrm{RBC}=$ red blood cells; $\mathrm{WBC}=$ white blood cells

$\mathbf{T}$ he demonstrated efficacy of the endovascular therapeutic approach to the occlusion of large vessels in the anterior circulation initiated a fundamental change in stroke therapy. This currently consists of the combination of thrombolysis with intravenous rtPA and mechanical endovascular thrombectomy (MT). ${ }^{1-6}$

With the increasing performance of MT to extract the occluding thrombus, more thromboembolic material has become available for further analysis. Several studies of thrombus composition

Received August 15, 2019; accepted after revision January 24, 2020.

From the Departments of Diagnostic and Interventional Radiology and Neuroradiology (J.G., I.W., M.F., C.M., A.R.) and Neuropathology (A.J.), and Clinic of Neurology (C.K., M.K.), University Hospital Essen, Essen, Germany; and Neuroradiological Centre (B.-J.G.), Clinic Hirslanden, Zurich, Switzerland. Please address correspondence to Juliane Goebel, MD, Department of Diagnostic and Interventional Radiology and Neuroradiology, University Hospital Essen, Hufelandstr 55, 45147 Essen, Germany; e-mail: Juliane.Goebel@uk-essen.de

Indicates article with supplemental on-line table.

Indicates article with supplemental on-line photos.

http://dx.doi.org/10.3174/ajnr.A6467 in acute ischemic stroke have been published, but a comparison of their data is difficult because of a lack of standardization, often with conflicting results. ${ }^{7,8}$ In addition, only a small number of thrombi have been investigated, often with no differentiation between fibrin and platelets or among the different subtypes of white blood cells (WBC). Therefore, the current study searched for the following: 1) histologic differences between the different stroke subtypes with regard to thrombus composition-that is, the proportions of fibrin, platelets, red blood cells (RBC), WBC, macrophages, lymphocytes, and granulocytes and the extent of thrombus fragmentation; and 2) correlations between thrombus composition and the parameters of the MT.

\section{MATERIALS AND METHODS}

Patients presenting at our center with acute ischemic stroke between December 2016 and March 2018 were recruited for MT and subsequent statistical analysis with the approval of the local 


\begin{tabular}{llcc}
\hline \multicolumn{1}{c}{ Antigen } & \multicolumn{1}{c}{ Source } & Pretreatment & Dilution \\
\hline CD68 (Ki-MIP) & Dr Klapper, Institute for Pathology, Kiel, Germany & None & $1: 10,000$ \\
CD45 (LCA) (mouse monoclonal, 2B11 + PD7/26) & Dako, Hamburg, Germany & Citrate & $1: 1000$ \\
\hline
\end{tabular}

Note:-LCA indicates leukocyte common antigen.

ethics committee of the medical faculty of the university Duisburg-Essen, Germany.

A total of 136 consecutive patients underwent MT during this period (On-line Fig 1). Patients from whom thrombotic material suitable for histopathologic analysis was obtained during MT were included in the further analysis. Patient characteristics were extracted from the electronic medical records. The subtypes of ischemic stroke were classified according to Hart. ${ }^{9}$

\section{Preinterventional Imaging}

Unenhanced brain CT and contrast-enhanced CTA of the supraaortic brain-supplying arteries (Somatom Definition Flash or Definition AS+ scanner; Siemens, Erlangen, Germany) were performed in all patients either in our center or in the referring hospital. All available unenhanced brain $\mathrm{CT}$ scans were retrospectively re-analyzed for the presence or absence of the dense artery sign, for thrombus density calculated as the average of 2 Hounsfield unit measurements in the occlusion indicated by CTA and/or the dense artery sign, and for early ischemic changes according to the ASPECTS in cases of middle cerebral artery ischemia. ${ }^{10}$

\section{Mechanical Thrombectomy}

MT using an aspiration catheter and/or a stent retriever was performed with the patient under general anesthesia in a biplanar DSA system (Allura Xper FD20; Philips Healthcare, Best, the Netherlands). By means of the Seldinger technique, a guiding catheter was inserted transfemorally, the large vessel proximal to the suspected occlusion was probed, and selective DSA was performed using Ultravist 300 as contrast agent (iopromide; Bayer HealthCare, Berlin, Germany). After identification of the arterial occlusion, a distal access or aspiration catheter was introduced coaxially (5F Sofia distal access catheter or 6F Sofia Plus aspiration catheter, MicroVention, Aliso Viejo, California; 3MAX/4MAX/5MAX or ACE 64 reperfusion catheter, Penumbra, Alameda, California) over a microwire (Traxcess14, MicroVention; Transend EX, Stryker, Kalamazoo, Michigan) and, if possible, docked onto the thrombus. Subsequently, direct contact thrombus aspiration was performed using a VacLok syringe (Merit Medical, South Jordan, Utah). If recanalization was not sufficient, the aspiration maneuver was repeated or, as an alternative, a stent retriever (Solitaire FR, Medtronic, Minneapolis, Minnesota; Trevo XP Provue System, Stryker; EmboTrap II Revascularization Device, Cerenovus, Galway, Ireland; Separator 3D System, Penumbra) was inserted, positioned in the thrombus, and 1 or multiple stent-retrieving maneuvers were then performed under constant aspiration. The thrombi were collected in a sterile manner, and those thrombi suitable for histopathologic analysis were immediately preserved in formalin. The records of the endovascular procedures were re-analyzed for interventional parameters, including the time interval between stroke onset and MT, the duration of MT, the endovascular devices/technique used, the number of retrieving maneuvers, and interventional complications. The achieved global recanalization success was quantified using the modified TICI (mTICI) scale. ${ }^{11}$

\section{Histology and Immunohistochemistry}

All investigations were performed on $1-\mu \mathrm{m}$ sections. Histochemical and immunohistochemical staining was used to determine the quantitative composition of the thrombi. The stains used were hematoxylin-eosin (H\&E) for RBC (appears reddish), Ladewig trichrome stain for fibrin deposits (appears brick red), Elastica van Gieson stain for collagen fibers (appears reddish), Von Kossa stain for calcifications (stains black, On-line Fig 2), naphthol AS-D chloroacetate stain for granulocytes (detected by their esterase content, On-line Fig 3), and Prussian blue staining for iron ions in siderophages. In addition, immunohistochemical staining for CD68 (positive for Ki-M1P, macrophages, and activated platelets, their size allowing exact quantification) and for $\mathrm{CD} 45$ (positive for leukocyte common antigen and lymphocytes) was performed (Online Fig 3). Pretreatments and antibody dilutions were performed as described in Table 1. In brief, the endogenous peroxidase activity was first blocked by incubating the sections in $3 \% \mathrm{H}_{2} \mathrm{O}_{2}$ in phosphate-buffered saline. This step was followed by a blocking step with $10 \%$ fetal calf serum in phosphate-buffered saline for 10 minutes at room temperature, followed by incubation with the primary antibody for 1 hour at room temperature. The sections were then incubated with the secondary antibody (biotin-labeled antibody). Because the sections were stained using the DakoAutostainer-Plus (Agilent Technologies, Santa Clara, California), the ZytoChemPlus horseradish peroxidase polymer system (mouse/rabbit) (REF:POLHRP-100, Dako, Hamburg, Germany) was used for detection. Cell nucleus counterstaining was performed with hematoxylin.

The stained sections were digitized using a Leica slide scanner (Leica Biosystems, Wetzlar, Germany). From the scanned files, either the whole thrombus area (H\&E for RBC quantification, Ladewig trichrome for fibrin quantification) or the whole thrombus area divided into subareas with an edge length of $1000 \mu \mathrm{m}$ (CD68 for monocytes/macrophages and activated platelets, CD45 for leukocytes) was extracted and analyzed using ImageJ software (National Institutes of Health, Bethesda, Maryland). ${ }^{12}$ After we adjusted the hue, saturation, and brightness, we adjusted the "color threshold" so that colored particles or colored areas could be determined using the "Analyze Particles" function in ImageJ. ${ }^{13}$ Regions with densely packed RBC or platelets and areas of dense fibrin deposition were included in the evaluation as coherent areas (On-line Fig 4).

The applied staining methods also allowed an age estimation of the thrombi. Because the Prussian blue stain, 
Table 2: Baseline characteristics and preinterventional imaging results of the studied patients $^{\mathrm{a}}$

\begin{tabular}{lc}
\hline \multicolumn{1}{c}{ Characteristics and Results } & \\
\hline Total No. of patients & 85 \\
Sex (No.) & 37 Men/48 women \\
Age (yr) & $72.0 \pm 12.9$ \\
Vascular risk factors (No.) (multiple choices possible) & \\
Atrial fibrillation & $50(58.8 \%)$ \\
Hypertension & $70(82.4 \%)$ \\
Diabetes & $24(28.2 \%)$ \\
High blood cholesterol levels & $53(62.4 \%)$ \\
Smoking & $19(22.4 \%)$ \\
Excessive alcohol intake & $1(1.2 \%)$ \\
Obesity & $7(8.2 \%)$ \\
Heart disease other than atrial fibrillation & $44(51.8 \%)$ \\
Patients with previous ischemic stroke (No.) & $18(21.2 \%)$ \\
Subtypes of ischemic stroke (No.) & \\
Major-risk source cardiogenic embolism & $51(60.0 \%)$ \\
Large-artery atherosclerotic stenosis & $16(18.8 \%)$ \\
ESUS & $17(20.0 \%)$ \\
Unusual (eg, dissection) & $1(1.2 \%)$ \\
Initial NIHSS score & $13(0-28)$ \\
Initial stroke imaging (No.) & \\
CT & 83 \\
DSA & 2 \\
Occlusion site (No.) & \\
Internal carotid artery (intracranial) & \\
MCA, M1 segment & $28(32.9 \%)$ \\
MCA, M2 segment & $36(42.4 \%)$ \\
Basilar artery & $10(11.8 \%)$ \\
Anterior cerebral artery & $9(10.6 \%)$ \\
Posterior cerebral artery & $1(1.2 \%)$ \\
Dense artery sign ( $n=71$ patients) & $1(1.2 \%)$ \\
Mean thrombus attenuation (HU) (67 patients) ${ }^{\mathrm{b}}$ & $43(60.6 \%)$ \\
Initial ASPECTS (in MCA ischemia; $n=64$ patients) & $58(41-149)$ \\
Patients given IV tPA (No.) & $10(3-10)$ \\
OTT ( $n=56$ patients) (min) & $52(61.2 \%)$ \\
\hline Note- & $172(96-782)$ \\
\hline
\end{tabular}

Note:-OTT indicates time interval between symptom onset and start of thrombectomy.

${ }^{a}$ Data are given as number (percent of total), mean \pm SD, or median (range).

${ }^{\mathrm{b}}$ Data were not available from all 85 patients (On-line Table 1).

indicating iron ions in siderophages, turns positive approximately 72 hours after coagulation, the absence of any blue coloration indicated a thrombus age of $<72$ hours. In addition, the intermingling of thrombi with collagen fibers, reddish in Elastica van Gieson staining, would argue for their reorganization, a finding that does not occur in fresh thrombi ( $<72$ hours) (On-line Fig 2). Furthermore, calcifications (visualized by the Von Kossa staining) are not found in fresh thrombi (On-line Fig 2).

The areas occupied by fibrin, platelets, RBC, and WBC (including macrophages, lymphocytes, and granulocytes) were quantified as percentages. Furthermore, we investigated the presence of siderophages, collagen fibers, calcifications, and the number of histologic fragments of the thrombi.

\section{Statistical Analysis}

Statistical analysis was performed with the SPSS software package (Version 25.0; IBM, Armonk, New York). Normal distribution was determined with the Kolmogorov-Smirnov test. Data are given as frequency counts and percentage values, medians and ranges, and means and SDs, as appropriate. The Kruskal-Wallis test was used to evaluate overall group differences between stroke subtypes in thrombus composition (fibrin, platelets, RBC, WBC, macrophages, lymphocytes, granulocytes, number of thrombus fragments). In case of significant overall group differences, pair-wise comparisons of stroke subtypes were performed with the Kruskal-Wallis 1-way ANOVA for multiple samples with a Bonferroni correction. To investigate a possible influence of thrombus composition on MT, we performed a KruskalWallis test (with post hoc Bonferroni correction for the second step of analysis) or a Spearman rank correlation analysis, as appropriate. Finally, a Mann-Whitney $U$ test was used to determine whether rtPA administration resulted in differences in fibrin content or fragmentation of the thrombi. A $P$ value $<.05$ was considered statistically significant.

\section{RESULTS \\ Patients, Preinterventional Imaging, and Interventional Parameters}

In 2 of the 136 initially recruited consecutive patients with acute ischemic stroke selected for MT, intravenous rtPA completely dissolved the occluding thrombi (On-line Fig 1). In the remaining 134 patients, MT failed in 25 patients (mTICI 0 in 16 patients, mTICI 1 in 2 patients, and mTICI $2 \mathrm{a}$ in 7 patients) and was successful in 109 patients (mTICI $2 \mathrm{~b}$ in 45 patients and mTICI 3 in 64 patients). In 48 patients, no evaluable thromboembolic material was extracted. Thrombi suitable for histopathologic analysis were retrieved from 86 patients, but 1 of these was excluded due to the use of a nonstandard recanalization technique. Thus 85 patients and their thrombi were included in this study.

Baseline characteristics, subtype of stroke, and preinterventional imaging results of the studied patients are shown in Table 2. Forty-five patients were directly admitted to our center as emergency patients (52.9\%), 33 patients $(38.8 \%)$ were referred to us by other hospitals, 5 patients developed an acute cerebral artery occlusion as in-patients in our hospital $(5.9 \%)$, and in 2 patients $(2.4 \%)$, artery occlusion was diagnosed during an angiography. The initial CT was not available for re-analysis in 11 of the patients transferred for MT from other hospitals to our center. Interventional and posttherapeutic parameters, including the duration of MT, global recanalization success, and the ultimately successful 
Table 3: Interventional parameters including revascularization rates and data on histopathologic thrombus composition ${ }^{\mathrm{a}}$

\begin{tabular}{|c|c|}
\hline Parameters & \\
\hline $\begin{array}{l}\text { Duration of thrombectomy (interval between first and last obtained DSA; } \\
\qquad n=83 \text { patients) (min) }\end{array}$ & $46(4-260)$ \\
\hline \multicolumn{2}{|l|}{ Achieved tissue reperfusion (No.) } \\
\hline $\mathrm{mTICl} 0$ & 5 (5.9\%) \\
\hline $\mathrm{mTICl} 1$ & $1(1.2 \%)$ \\
\hline $\mathrm{mTICl} 2 \mathrm{a}$ & $2(2.4 \%)$ \\
\hline $\mathrm{mTICl} 2 \mathrm{~b}$ & $34(40.0 \%)$ \\
\hline $\mathrm{mTICl} 3$ & $43(50.6 \%)$ \\
\hline \multicolumn{2}{|l|}{ Ultimately successful recanalization technique (No.) } \\
\hline Aspiration (number of passes, median and range) 2 (1-8) & $47(55.3 \%)$ \\
\hline Stent retrieval (number of passes, median and range) $2(1-5)$ & $23(27.1 \%)$ \\
\hline $\begin{array}{l}\text { Combined aspiration and stent retrieval (number of passes, median and } \\
\text { range) } 3(2-8)\end{array}$ & $15(17.6 \%)$ \\
\hline Occurrence of endovascular complications (No.) & $19(22.4 \%)$ \\
\hline Occurrence of symptomatic hemorrhage (No.) & $2(2.4 \%)$ \\
\hline ASPECTS $24 \mathrm{hr}$ postthrombectomy $\left(n=73\right.$ patients) ${ }^{\mathrm{b}}$ & $8(0-10)$ \\
\hline NIHSS score at 2-14 days postthrombectomy $\left(n=66\right.$ patients) ${ }^{b}$ & $3(0-21)$ \\
\hline \multicolumn{2}{|l|}{ Thrombus components (\% from thrombus profile) } \\
\hline Fibrin & $40.9(2.8-68.2)$ \\
\hline Platelets & $16.1(1.9-81.1)$ \\
\hline $\mathrm{RBC}$ & $41.7(1.2-89.9)$ \\
\hline WBC & $2.6(0.4-8.5)$ \\
\hline Macrophages & $0.7(0.0-5.2)$ \\
\hline Lymphocytes & $0.3(0.0-1.7)$ \\
\hline Granulocytes & $1.5(0.0-7.1)$ \\
\hline No. of thrombus fragments & $8(2-47)$ \\
\hline
\end{tabular}

Note:- DSA indicates digital subtraction angriography.

${ }^{a}$ Data are given as number and percentage or median and range.

${ }^{b}$ Data were not available in all 85 patients (On-line Table 1).
Mechanical Thrombectomy. Thrombi retrieved from patients who had received intravenous $\mathrm{rtPA}$ contained significantly less fibrin $(38.4 \%, 2.8 \%-$ $66.4 \%)$ than those from patients without rtPA administration (42.4\%, 20.1\%-68.2\%; $P=.027$ ), whereas no correlation was found between rtPA administration and the extent of thrombus fragmentation. With regard to the ultimately successful recanalization technique, thrombi that could only be retrieved using a combination of contact aspiration and stent retrieval had a significantly smaller proportion of platelets $(11.5 \%, 2.5 \%-35.3 \%)$ than those that were removed by stent retrieval alone $(18.4 \%, 5.2 \%-39.7 \% ; P=$ .034 ; Fig 2). Thrombi that were only extractable using the combination technique had a significantly higher number of fragments $(13,3-40)$ than thrombi extractable by contact aspiration (8, $2-35 ; P=.021)$ or stent retrieval alone $(7,2-47 ; P=.006 ;$ Fig 2$)$. There was a weak correlation between the number of thrombus fragments and the required number of retrieving maneuvers ( $r=0.365, P=.001)$, procedural time recanalization technique with the number of retrieval maneuvers, are given in Table 3.

\section{Histologic Thrombus Composition}

The quantitative contributions of fibrin, platelets, RBC, and WBC to the investigated thrombi are shown in Fig 1. The percentage distribution of the different thrombus components is shown in Table 3. Because blue staining for iron ions in siderophages was always negative and neither calcifications nor collagen fibers were detected, all thrombi were classified as fresh (ie, $<3$ days old).

Subtypes of Stroke. Because there was just 1 patient with an unusual stroke subtype, statistical comparisons were possible only in cardioembolic, large-artery atherosclerotic stenosis (LAA), and embolic stroke of undetermined source (ESUS) subtypes.

The proportion of macrophages was significantly higher in cardioembolic thrombi $(0.9 \%, 0.1 \%-3.3 \%)$ than in LAA $(0.3 \%$, $0.1 \%-3.8 \% ; P=.021)$ or ESUS thrombi $(0.4 \%, 0.0 \%-5.2 \% ; P=$ .037 ; Fig 2). Cardioembolic thrombi contained a higher proportion of platelets $(19.1 \%, 3.6 \%-81.1 \%)$ than LAA thrombi $(10.3 \%$, $2.3 \%-25.1 \% ; P=.003)$ and showed a tendency toward a higher platelet proportion compared with ESUS thrombi $(12.2 \%, 1.9 \%-$ $39.7 \%$; $P=.099$; Fig 2). LAA and ESUS thrombi did not differ significantly with regard to platelet or macrophage content, nor were other differences in thrombus composition found between stroke subtypes.
( $r=0.356, P=.001)$, and percentage of lymphocytes in the thrombi $(r=0.229, P=.035)$.

\section{DISCUSSION}

This study showed, first, that cardioembolic thrombi had a higher percentage of macrophages and a trend toward a higher percentage of platelets than thrombi of other stroke subtypes and, second, thrombi presenting as severely fragmented for histologic work-up had a higher lymphocyte content and often required the combined application of contact aspiration and stent retrieval, as well as more retrieving maneuvers and longer procedure times for their removal.

Regarding stroke etiology, we observed a higher percentage of macrophages and a trend toward a higher percentage of platelets in cardioembolic thrombi than in LAA or ESUS thrombi, while there were no such differences between LAA and ESUS strokesubtype thrombi. We interpreted this finding as an indication that ESUS represents an embolism from an atherosclerotic plaque rather than from an undetermined cardiac embolic source. This is in line with the results of Boeckh-Behrens et $\mathrm{al}^{14}$ and Sporns et $\mathrm{al}^{15}$ who reported higher WBC and fibrin/platelet content in cardioembolic thrombi, and with the findings of Maekawa et $\mathrm{al}^{16}$ who reported an association between fibrin-rich thrombi and the cardioembolic stroke subtype, though the latter did not differentiate between fibrin and thrombocytes. The few published studies with small numbers of examined thrombi did not find any association between thrombus composition and stroke subtype. ${ }^{17-19}$ In addition, contrary to our results, Kim et $\mathrm{al}^{20}$ reported a higher 

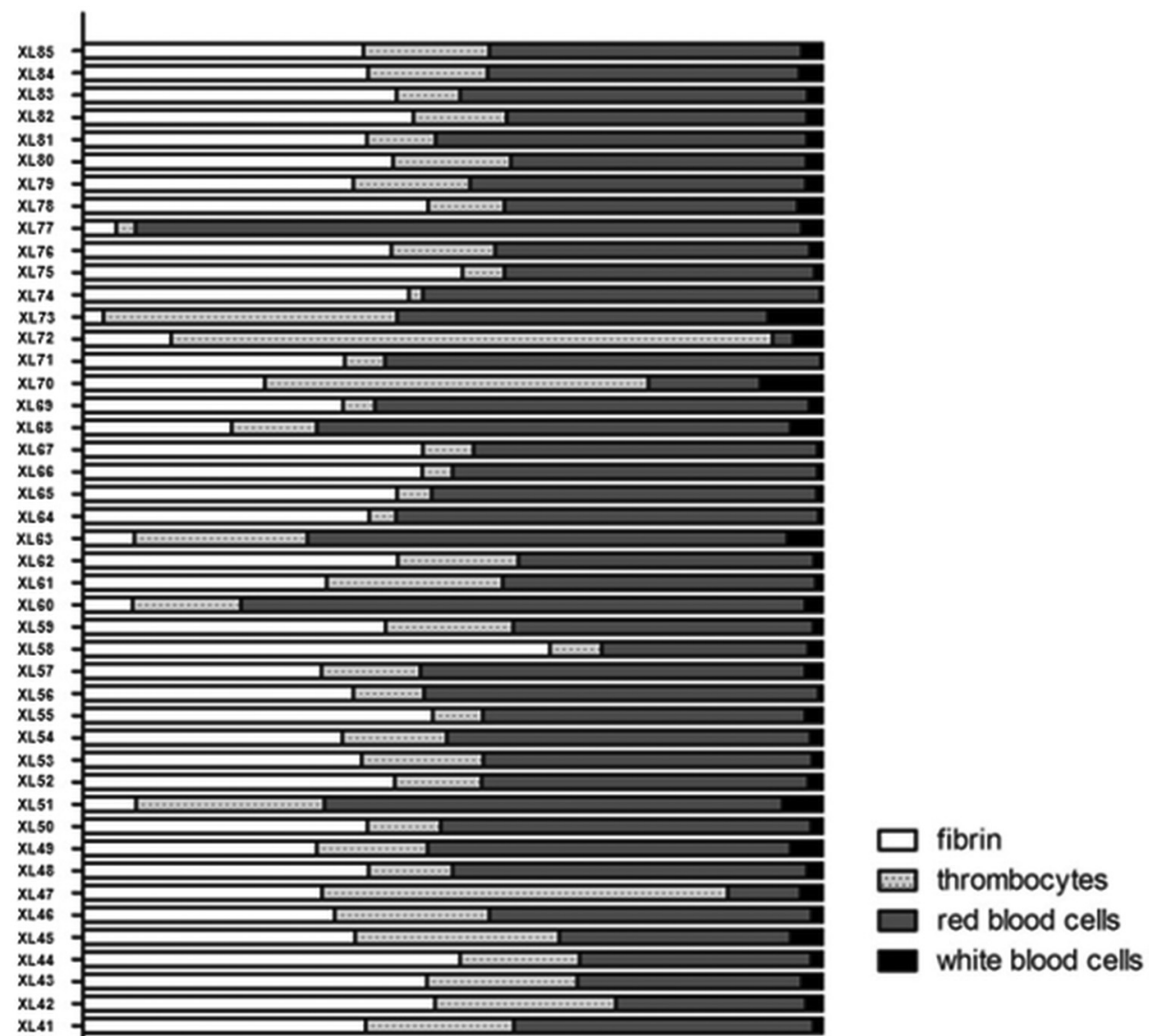

XL40 -

XL59

$\mathrm{XL38}$

$\mathrm{XLS7}$

$\mathrm{XL36}$

$X \mathrm{LS}$

$\mathrm{XL34}$

$\mathrm{XL32}$

$\mathrm{x}: 31$

$\mathrm{X} 130$

$x เ 28$

$x เ 27$

$\mathrm{x}: 26$

$x+24$

$x: 22$

$\mathrm{X}: 21$

$x<20$

$\mathrm{XL19}$

$\mathrm{xL17}$

$\mathrm{XL} 16$ 20

$\mathrm{XL15}$

XL14

$x: 12$

$\mathrm{XL11}$

$\mathrm{x} 609$

$\times 109$
$\times 108$

$\mathrm{x} 107$

$\times 105$

$x$ xLO5

$\mathrm{x} \times 03$

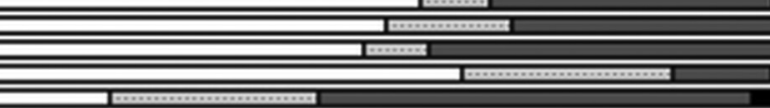

=

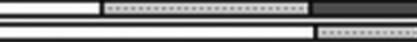

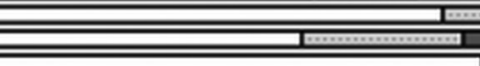

$=$

$\overline{100}$

2. 2

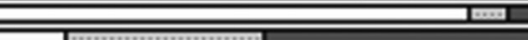

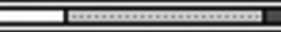
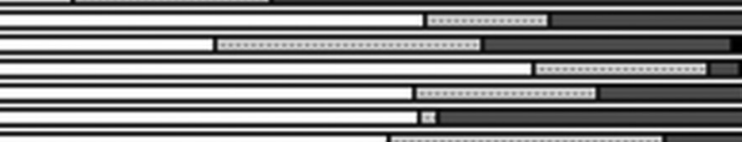

(2)

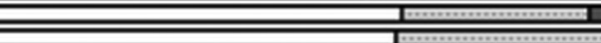

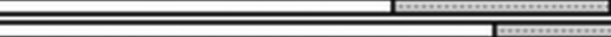
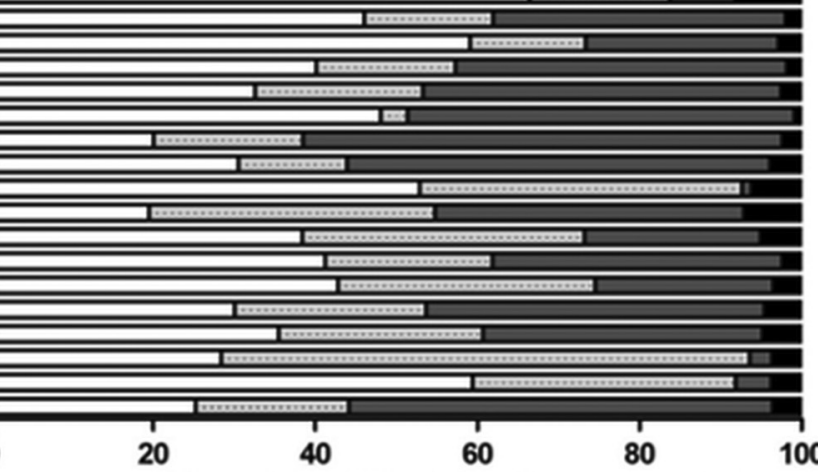

FIG 1. Percentages of the different clot components. Each investigated thrombus is shown in its quantitative composition of fibrin, platelets, red blood cells, and white blood cells (including macrophages, leukocytes, and granulocytes). 

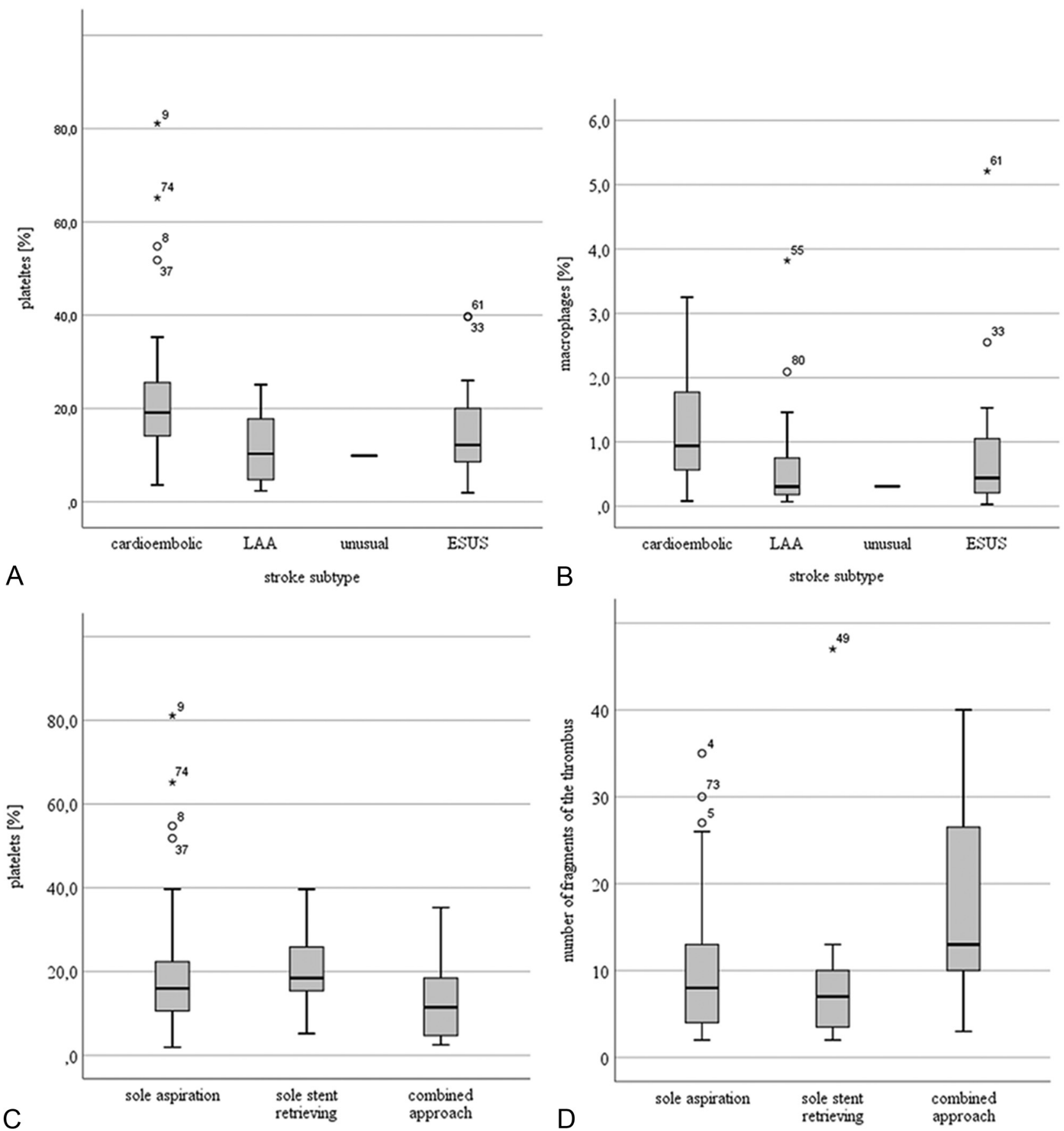

FIG 2. Differences between the different stroke subtypes in the proportions of platelets and macrophages (subunits $A$ and $B$ ) and differences between the individual thrombi in the proportions of platelets and the number of fragments that were ultimately retrieved by contact aspiration alone, stent retrieval alone, or a combination of the 2 techniques (subunits $C$ and $D$ ).

proportion of RBC and a lower proportion of fibrin in cardioembolic thrombi than in LAA thrombi. Nevertheless, because only 8 LAA thrombi were included in their study, its validity might be questionable.

Most interesting, we found significant relationships between thrombus composition and parameters of the MT procedure as described above. This finding raises the following the question: Was, for example, increased thrombus fragmentation caused by the MT procedure itself, because thrombus fragmentation with peripheral embolization is a known complication of MT, or was the tendency of some thrombi to fragment due to their composition ${ }^{21}$ It is possible that thrombi prone to fragmentation are more difficult to remove by MT and require more retrieving maneuvers consecutively, resulting in a longer procedural time. The additionally observed relationship between lymphocyte content and the degree of thrombus fragmentation supports the assumption of a lymphocyte-rich subgroup of thrombi that are susceptible to fragmentation. This would support the hypothesis that the greater extent of thrombus fragmentation was not exclusively due to the recanalization procedure but was, to a certain 
degree, an inherent tendency for these thrombi to fragment. Because lymphocytes are known to play a role in thrombus formation, ${ }^{19}$ might they not conceivably also make the thrombus more prone to fragmentation? Our findings are partly in line with those of Kaesmacher et $\mathrm{al}^{22}$ who reported an association between a higher content of neutrophil granulocytes and increased periprocedural thrombus fragmentation but found no relationship between thrombus stability and the overall WBC content. We found that thrombi that had required the combined approach of contact aspiration and stent retrieval for their extraction had a lower proportion of platelets than those that could be extracted by stent retrieval alone. We are not quite sure how to interpret this finding, and the results of other studies on this topic are of no assistance because they did not differentiate between platelets and fibrin. ${ }^{16,17,22-24}$

Our study has several limitations. The first of these is a selection bias. We were obviously able to examine only those thrombi that could be retrieved, thus excluding thrombectomy-resistant thrombi. A second limitation is the small number of retrieved thrombi and the skewed distribution between the different stroke subtypes. Finally, we had no information on the prevalence of primarily fragmented thrombi because the preinterventional imaging was unenhanced brain CT and CTA and we directly probed the large vessel proximal to the suspected occlusion during MT.

\section{CONCLUSIONS}

We found that cardioembolic thrombi had a higher percentage of macrophages and a tendency toward a higher percentage of platelets than thrombi of the LAA or ESUS stroke subtype. We interpreted this finding as an indication that ESUS represents embolism from an atherosclerotic plaque rather than from an undetermined cardiac embolic source. Moreover, we observed an association between the extent of thrombus fragmentation and both a more challenging mechanical thrombectomy and a higher lymphocyte content. Thus, thrombus fragmentation might be caused not only by the recanalization procedure but might also be due to an inherent feature of lymphocyte-rich and difficult-to-retrieve thrombi.

\section{ACKNOWLEDGMENTS}

We thank Dr Klapper (Institute for Pathology, Kiel, Germany) for the kind donation of antibodies against CD68 (Ki-M1P) and Nicole Macha for excellent technical assistance.

Disclosures: Isabel Wanke-UNRELATED: Consultancy: Stryker Osteonics SA, Comments: Steering Committee for Neuroform Atlas Stent for Intracranial Aneurysm Treatment (ATLAS EU PMCF, microstent), less than $€ 800$ per year; Payment for Lectures Including Service on Speakers Bureaus: Bayer Health, Comments: 2 lectures per year $(2 \times$ $€ 1000)$. Christoph Moenninghoff_UNRELATED: Payment for Lectures Including Service on Speakers Bureaus: Teva Pharmaceutical Industries, Comments: payment for 1 lecture; Payment for Manuscript Preparation: Bayer Pharmaceuticals, Comments: payment for 1 MRA image set preparation; Travel/Accommodations/Meeting Expenses Unrelated to Activities Listed: MicroVention, Stryker, Comments: several neurointerventional meetings. Alexander Radbruch—UNRELATED: Other. Study support from Guerbet and Bayer, personal fees from Bayer, Guerbet, Siemens, Sanofi, GE Healthcare, and Bracco.

\section{REFERENCES}

1. Berkhemer OA, Fransen PS, Beumer D, et al; MR CLEAN Investigators. A randomized trial of intraarterial treatment for acute ischemic stroke. $N$ Engl J Med 2015;372:11-20 CrossRef Medline

2. Goyal M, Demchuk AM, Menon BK, et al; ESCAPE Trial Investigators. Randomized assessment of rapid endovascular treatment of ischemic stroke. $N$ Engl J Med 2015;372:1019-30 CrossRef Medline

3. Campbell BC, Mitchell PJ, Kleinig TJ, et al; EXTEND-IA Investigators. Endovascular therapy for ischemic stroke with perfusion-imaging selection. $N$ Engl J Med 2015;372:1009-18 CrossRef Medline

4. Saver JL, Goyal M, Bonafe A, et al; SWIFT PRIME Investigators. Stent-retriever thrombectomy after intravenous t-PA vs. T-PA alone in stroke. $N$ Engl J Med 2015;372:2285-95 CrossRef Medline

5. Jovin TG, Chamorro A, Cobo E, et al; REVASCAT Trial Investigators. Thrombectomy within 8 hours after symptom onset in ischemic stroke. N Engl J Med 2015;372:2296-2306 CrossRef Medline

6. Goyal M, Menon BK, van Zwam WH, et al; HERMES Collaborators. Endovascular thrombectomy after large-vessel ischaemic stroke: a meta-analysis of individual patient data from five randomised trials. Lancet 2016;387:1723-31 CrossRef Medline

7. Brinjikji W, Duffy S, Burrows A, et al. Correlation of imaging and histopathology of thrombi in acute ischemic stroke with etiology and outcome: a systematic review. J Neurointerv Surg 2017;9:529-34 CrossRef Medline

8. De Meyer SF, Andersson T, Baxter B, et al; Clot Summit Group. Analyses of thrombi in acute ischemic stroke: a consensus statement on current knowledge and future directions. Int J Stroke 2017;12:606-14 CrossRef Medline

9. Hart RG, Diener HC, Coutts SB, et al; Cryptogenic Stroke/ESUS International Working Group. Embolic strokes of undetermined source: the case for a new clinical construct. Lancet Neurol 2014;13:429-38 CrossRef Medline

10. Barber PA, Demchuk AM, Zhang J, et al. Validity and reliability of a quantitative computed tomography score in predicting outcome of hyperacute stroke before thrombolytic therapy: ASPECTS study group: Alberta Stroke Programme Early CT Score. Lancet 2000;355: 1670-74 CrossRef Medline

11. Zaidat OO, Yoo AJ, Khatri P, et al; STIR Thrombolysis in Cerebral Infarction (TICI) Task Force. Recommendations on angiographic revascularization grading standards for acute ischemic stroke: a consensus statement. Stroke 2013;44:2650-63 CrossRef Medline

12. Schindelin J, Rueden CT, Hiner MC, et al. The ImageJ ecosystem: an open platform for biomedical image analysis. Mol Reprod Dev 2015;82:518-29 CrossRef Medline

13. Voigt D, Scheidt U, Derfuss T, et al. Expression of the antioxidative enzyme peroxiredoxin 2 in multiple sclerosis lesions in relation to inflammation. Int J Mol Sci 2017;18 CrossRef Medline

14. Boeckh-Behrens T, Kleine JF, Zimmer C, et al. Thrombus histology suggests cardioembolic cause in cryptogenic stroke. Stroke 2016;47:1864-71 CrossRef Medline

15. Sporns PB, Hanning U, Schwindt W, et al. Ischemic stroke: what does the histological composition tell us about the origin of the thrombus? Stroke 2017;48:2206-10 CrossRef Medline

16. Maekawa K, Shibata M, Nakajima H, et al. Erythrocyte-rich thrombus is associated with reduced number of maneuvers and procedure time in patients with acute ischemic stroke undergoing mechanical thrombectomy. Cerebrovasc Dis Extra 2018;8:39-49 CrossRef Medline

17. Liebeskind DS, Sanossian N, Yong WH, et al. CT and MRI early vessel signs reflect clot composition in acute stroke. Stroke 2011;42:1237-43 CrossRef Medline

18. Simons N, Mitchell P, Dowling R, et al. Thrombus composition in acute ischemic stroke: A histopathological study of thrombus extracted by endovascular retrieval. J Neuroradiol 2015;42:86-92 CrossRef Medline

19. Schuhmann MK, Gunreben I, Kleinschnitz C, et al. Immuno-histochemical analysis of cerebral thrombi retrieved by mechanical 
thrombectomy from patients with acute ischemic stroke. Int J Mol Sci 2016;17:298 CrossRef Medline

20. Kim SK, Yoon W, Kim TS, et al. Histologic analysis of retrieved clots in acute ischemic stroke: correlation with stroke etiology and gradient-echo MRI. AJNR Am J Neuroradiol 2015;36:1756-62 CrossRef Medline

21. Gralla J, Schroth G, Remonda L, et al. Mechanical thrombectomy for acute ischemic stroke: thrombus-device interaction, efficiency, and complications in vivo. Stroke 2006;37:3019-24 CrossRef Medline
22. Kaesmacher J, Boeckh-Behrens T, Simon S, et al. Risk of thrombus fragmentation during endovascular stroke treatment. AJNR Am J Neuroradiol 2017;38:991-98 CrossRef Medline

23. Hashimoto T, Hayakawa M, Funatsu N, et al. Histopathologic analysis of retrieved thrombi associated with successful reperfusion after acute stroke thrombectomy. Stroke 2016;47:3035-37 CrossRef Medline

24. Boeckh-Behrens T, Schubert M, Forschler A, et al. The impact of histological clot composition in embolic stroke. Clin Neuroradiol 2016; 26:189-97 CrossRef Medline 